\title{
The protocol of a randomized controlled trial for playgroup mothers: Reminder on Food, Relaxation, Exercise, and Support for Health (REFRESH) Program
}

Sarojini MDR Monteiro ${ }^{1,2^{*}}$, Jonine Jancey ${ }^{1,2}$, Peter Howat ${ }^{1,2}$, Sharyn Burns ${ }^{1}$, Carlie Jones ${ }^{1,2}$, Satvinder S Dhaliwal ${ }^{1,2}$, Alexandra McManus ${ }^{3}$, Andrew P Hills ${ }^{4}$ and Annie S Anderson ${ }^{5}$

\begin{abstract}
Background: Mother's physical activity levels are relatively low, while their energy consumption is generally high resulting in 58\% of Australian women over the age of 18 years being overweight or obese. This study aims to confirm if a low-cost, accessible playgroup based intervention program can improve the dietary and physical activity behaviours of mothers with young children.

Methods/Design: The current study is a randomized controlled trial lifestyle (nutrition and physical activity) intervention for mothers with children aged between 0 to 5 years attending playgroups in Perth, Western Australia. Nine-hundred participants will be recruited and randomly assigned to the intervention $(n=450)$ and control $(n=450)$ groups. The study is based on the Social Cognitive Theory (SCT) and the Transtheoretical Model (TTM), and the Precede-Proceed Framework incorporating goal setting, motivational interviewing, social support and self-efficacy. The six month intervention will include multiple strategies and resources to ensure the engagement and retention of participants. The main strategy is home based and will include a specially designed booklet with dietary and physical activity information, a muscle strength and flexibility exercise chart, a nutrition label reading shopping list and menu planner. The home based strategy will be supported by face-to-face dietary and physical activity workshops in the playgroup setting, posted and emailed bi-monthly newsletters, and monthly Short Message Service (SMS) reminders via mobile phones. Participants in the control group receive no intervention materials. Outcome measures will be assessed using data that will be collected at baseline, six months and 12 months from participants in the control and intervention groups.
\end{abstract}

Discussion: This trial will add to the evidence base on the recruitment, retention and the impact of community based dietary and physical activity interventions for mothers with young children.

Trial Registration: Australian and New Zealand Clinical Trials Registry ACTRN12609000735257

\section{Background}

Overweight and obesity are important public health concerns. The percentage of Australian women of childbearing age that are overweight or obese has significantly increased over the past decade. In $2007,44 \%$ of Australian women aged between 25 and 34 years were overweight or obese compared to only $26 \%$ in 1995 [1].

\footnotetext{
* Correspondence: S.Monteiro@curtin.edu.au

'Western Australian Centre for Health Promotion Research, School of Public Health, Curtin University, Western Australia, Australia

Full list of author information is available at the end of the article
}

Childbearing aged women are an important target group for dietary and physical activity interventions as they are at an increased risk of long-term overweight and obesity [2]. Women's increased risk of overweight and obesity after their first and subsequent pregnancies is associated with overweight or obesity prior to pregnancy $[3,4]$, gestational weight gain above the recommended guidelines [5,6], failure to lose gestational weight in an appreciable timeframe or excessive postpartum weight retention [7] and interpregnancy weight gain [8].

\section{C) Biomed Central}


Overweight and obese childbearing aged women appear to have a disproportionate risk of maternal, intrapartum, peripartum, neonatal, and postpartum complications $[9,10]$. If this weight gain continues after childbearing, women will be at increased risk of obesity related chronic conditions such as type II diabetes, high blood pressure, dyslipidaemia, cardiovascular disease and the risk of several major cancers [10]. In addition, maternal obesity may have deleterious effects on the neonate such as macrosomia, increased risk of a range of structural anomalies and of still birth [11].

Research indicates that the mechanisms for interpregnancy and 12 months postpartum weight gain can be due to a range of factors such as lack of nutrition knowledge [12], poor dietary habits and physical inactivity $[13,14]$. For example, research shows that $96 \%$ of females aged $25-$ 34 and $94 \%$ of females aged 35-44 consume inadequate fruit or vegetables when compared to the Australian dietary guidelines [1]. Furthermore, despite the known health benefits of physical activity, 30\% of women aged between 24 and 34 do not do any exercise, while $44 \%$ participate in low intensity activity [1].

The barriers to mothers adopting the recommended physical activity behaviours include lack of social support, lack of time, lack of energy and motivation, procrastination, lack of self-efficacy and childcare and financial constraints $[15,16]$. The influences on eating habits include convenience, cost, lifestyle preferences, confusion around food messages, nutrition knowledge and environmental factors [17]. Furthermore, common postpartum physical symptoms such as fatigue, headaches, nausea, backache and urinary or bowel problems can inhibit mothers following a healthy diet and physical activity plan [18].

Mothers are an important group within the family unit as they are generally the primary caregiver and help to shape the attitudes and behaviour of their children with respect to food and physical activity. Overweight and obese children are twice as likely to become overweight and obese adults when compared to normal weight children [19]. Mothers can prevent children from becoming overweight and obese as they play a major role in determining the family mealtime environment, and managing the amount and type of food available [20]. Thus, efforts to interrupt this cycle of obesity by targeting interventions at mothers are vital from both a public health perspective. Dietary and physical activity interventions could provide benefits to the mother, her future pregnancies and subsequent generations from becoming overweight and obese [21].

Currently, there are few studies that have reported the effectiveness of behavioural interventions designed to improve physical activity and dietary behaviours [22-26] in mothers with young children. These studies have included small samples and have incorporated limited evaluation measures [23-25], even though the evidence suggests that after childbirth mothers are ready to change behaviours associated with overweight and obesity $[27,28]$.

This paper describes the protocol of a randomized controlled trial to improve the physical activity and nutrition behaviours of mothers with young children (between 0 and 5 years of age) attending playgroups.

\section{Methods/Design Study design}

The study is a community based 12 month randomized controlled trial. The study is designed according to the recommendations of the CONSORT statement for randomized trials of nonpharmacologic treatment [29].

The REFRESH study will be conducted over three years (Figure 1). The first year will include formative research, development of the evaluation framework and the intervention. In the second year, participants will be recruited, the intervention will be implemented and data will be collected from participants. The final year will include data collection, data analysis and review of the intervention.

\section{Study aim}

The REFRESH study aims to evaluate the effect of a six month physical activity and nutrition randomized controlled trial for mothers with young children attending playgroups in Perth, Western Australia (WA).

The REFRESH program will focus on behaviour change to meet the Australian physical activity guidelines, by encouraging increased in levels of vigorous, and moderate physical activity, the number of steps taken each day and muscle strength exercises [30,31]. The REFRESH program will also aim to encourage behaviour change to meet the Australian dietary guidelines (improve nutritional intake by increasing fruit, vegetable and fibre intake and decreasing fat and added sugar intake) [32].

\section{Settings}

Playgroups in Australia are informal regular community groups that are set up for babies, toddlers and pre-school children ( 0 to 5 years). The purpose of a playgroup is to encourage play among children to enhance their social, emotional, physical and intellectual development. Parents and carers also find it a valuable resource as they help establish support networks. Playgroups are run by volunteer parents and carers who get together once a week for a couple of hours. They are held at a variety of venues such as libraries, child and maternal health centres, church halls, kindergartens and schools. Playgroups are supported by National and State organisations [33]. The REFRESH project will be conducted in collaboration with Playgroup WA Inc. [34], as the playgroup will be used as the setting to recruit mothers and implement the project. 


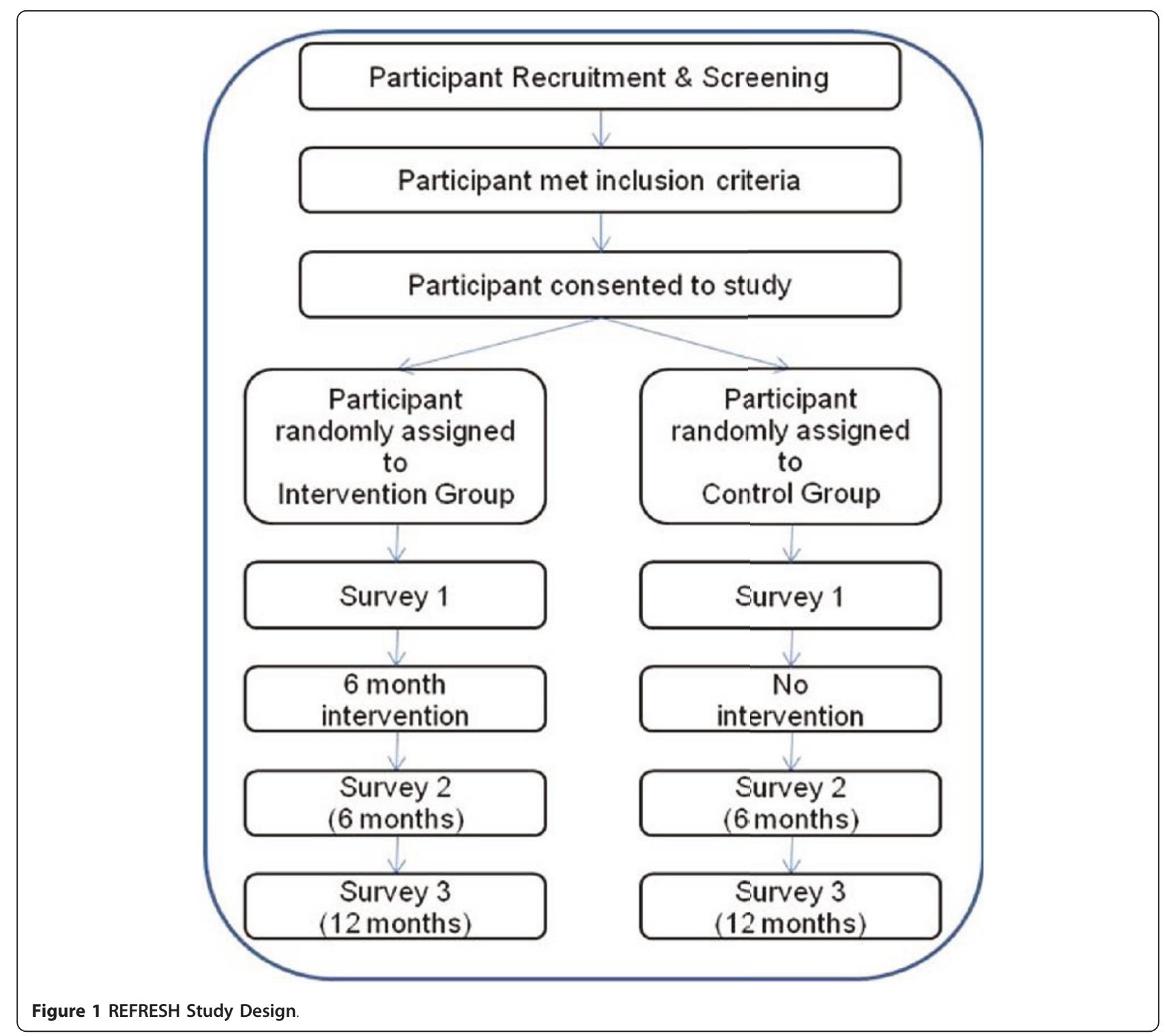

\section{Recruitment and randomisation process}

A stratified random sampling procedure will be adopted to recruit participants from 560 playgroups embedded in 106 suburbs (neighbourhoods) within the Perth metropolitan area. Stratification will be conducted by suburb geographical location and Socio-Economic Indexes For Area (SEIFA) scores. SEIFA scores are values derived from income, education level, employment status and skill level [35]. The suburbs will then be randomly assigned to either the intervention group or the control group using a table of random numbers. Control and intervention group suburbs will be arbitrarily matched for low and medium levels of socio-economic status based on the SEIFA scores. The senior Playgroup WA Inc. staff will make phone calls to all registered playgroup leaders, explain the REFRESH project and obtain permission for project staff to contact the playgroup. Project staff will visit the playgroup to further explain the project, obtain consent and allocate participants to the intervention or control group. Intervention group participants will also complete the Physical Activity Readiness Questionnaire [36] and provide a medical certificate if deemed necessary before commencing the program.

\section{Inclusion criteria}

Study participants will need to be: (a) women aged 18 or over registered with Playgroup WA Inc.; b) have a child between 0 to 5 years; (c) healthy to the extent that participation in a low-stress physical activity program would not place them at risk; (d) not taken part in any research that involves physical activity or nutrition within the last five years; and (e) not on a special diet. 


\section{Sample size determination}

In order to detect a $20 \%$ difference in physical activity at $80 \%$ power and 5\% level of significance, sample size of 310 mothers of young children are required at the 6-months post-intervention survey in each of the intervention and control groups. A small effect size (0.2) [37] is assumed for studies on behavioural effects due to the influence of extraneous variables and the subtleties of human performance. Allowing for an attrition rate of 30\%, 900 mothers of young children will be recruited into the study. Sample size calculations were determined using Power Analysis and Sample Size software [38].

\section{Data collection}

Process data will be collected during the implementation of the intervention. The playgroup is a novel setting for the recruitment and delivery of health promotion interventions for mothers with young children. Therefore, the process evaluation will be a key component of the program's evaluation. This will be conducted with both the participants and the project staff, providing two perspectives on the program delivery and content.

Outcome data will be collected at baseline, six months and 12 months. At baseline control group participants will be hand delivered a self-completion questionnaire at the playgroup along with a self-addressed envelope and measuring tape to record waist and hip measurements. The intervention group will be provided all of the above and a pedometer to record the number of steps taken each day. At six months the control group participants will be hand delivered a self-completion questionnaire at the playgroup along with a self-addressed envelope. The intervention group will be provided all of the above and a pedometer to record the number of steps taken each day. At 12 months the control and intervention group participants will be posted a self-completion questionnaire with a selfaddressed envelope.

\section{Blinding}

It is not possible to blind study project staff to the randomisation process, however, the participants will be blinded as to whether they are in the study or control group. The assessor will be blinded until the comparative data analysis is conducted. Participants will be given codes when recruited and these codes will be used throughout the implementation of the study. The participant codes will be revealed only at the six and 12 month comparative data analysis.

\section{Statistical analysis}

Data collected will be coded and analysed using the Statistical Package for the Social Sciences computer statistical software [39]. Descriptive statistics will first be used to summarise participants' demographic and health characteristics. For the hierarchical data (repeated measurements of individuals) collected over the one-year observational period, multi-level repeated measures analyses and multivariate logistic regression analyses will be used extensively in the statistical analyses.

\section{Intervention group}

To facilitate the development of the intervention and to ensure adherence to its timeline, the implementation of the intervention will be organised into four stages.

\section{Stage 1}

Intervention development A literature review of nutrition and physical activity community based interventions for mothers with young children, pregnant and postpartum women has been conducted and will be continuously updated. Relevant behaviour change theories reviewed including the Social Cognitive Theory (SCT) [40], Transtheoretical Model (TTM) [41] and motivational interviewing [42] will support the development of a multi-strategy intervention [43]. Previous qualitative data obtained from Perth playgroup mothers will be used to ascertain the barriers and facilitators to healthy eating and being physically active, as well as their preferred intervention strategies [44]. The Precede-Proceed model will be used to organise the behaviour change theories and formative research data into an appropriate nutrition and physical activity behaviour change program $[45,46]$.

\section{Stage 2}

Recruitment of staff The program will be staffed by Health Science graduates, who will deliver the face-to-face workshop styled information and skill building sessions. Recruitment of the health promotion, nutrition and sport science graduates will be conducted via local universities and relevant professional associations.

\section{Stage 3}

Staff training Staff will receive intensive training on the application of the Australian dietary and physical activity guidelines [31,32], and behaviour change theories including motivational interviewing. They will receive a comprehensive training manual on the delivery of the face-to-face workshop sessions. The staff will also receive ongoing support via email and phone by an accredited dietician, human movement specialist, health promotion specialist and the project coordinator.

\section{Stage 4}

Delivery of intervention in playgroup settings The intervention will be delivered over six months. Interventions that aim to address multiple risk factors such as nutrition and physical activity show more positive outcomes when multiple intervention strategies are used to reach the target audience $[43,47]$. Hence the intervention group participants will receive four strategies: face-to-face workshop information and skill development session; mailed or emailed newsletters; SMS reminders on the 
main messages of the REFRESH program; and a homebased component.

Delivery of face-to-face workshops The intervention group participants will receive six workshop sessions over six months (one session a month). Each session will be conducted for 30 minutes by project staff during the playgroup session at the playgroup venue. Workshops will focus on enhancing knowledge, attitudes and skills to enable informed decision making about nutrition and physical activity behaviours (Table 1).

Delivery of newsletters The intervention group participants will receive six newsletters via post or email over six months (one newsletter a month, one week after each face-to-face workshop session). The newsletters will be in an informal format and will contain myth dispelling information on nutrition and physical activity.
Delivery of SMS reminders The intervention group participants will receive 18 SMS reminders via mobile phones over six months (reminders to attend the faceto-face workshop sessions and nutrition and physical activity motivating messages).

Delivery of home based component The intervention group participants will receive home based resources at each of the face-to-face workshop sessions to support the content of the REFRESH program. The home based components will include a specially tailored program booklet, pedometer, menu planner fridge magnet, a shopping list with food label reading tips, a muscle strength and flexibility exercise chart fridge magnet, a physical activity diary and an 'extra' food record sheet. The workshops will offer an opportunity for these resources to be explained and for questions to be answered.

Table 1 REFRESH Intervention

\begin{tabular}{|c|c|c|}
\hline Session (Week) & Session Details & Participant resources/interactive activities \\
\hline 1 (Week 1) & $\begin{array}{l}\text { 1. Introduction to Refresh Program } \\
\text { 2. Overview of healthy eating and being physically active } \\
\text { 3. Focus nutrition: fruits, vegetables and water } \\
\text { - Guidelines } \\
\text { - Benefits/barriers/overcoming barriers }\end{array}$ & $\begin{array}{l}\text { 1. Resources: } \\
\text { - Program booklet } \\
\text { - Healthy recipe booklet } \\
\text { - Session one information summary pamphlet } \\
\text { 2. Interactive activity: } \\
\text { - Determine participant program needs }\end{array}$ \\
\hline 2 (Week 5) & $\begin{array}{l}\text { 1. Focus behaviour change: } \\
\text { - Stages of change } \\
\text { - Goal setting: long and short term goals } \\
\text { 2. Focus physical activity: aerobic } \\
\text { - Guidelines } \\
\text { - Benefits/barriers/overcoming barriers } \\
\text { 3. Focus nutrition: five food groups and 'extra' foods } \\
\text { - Guidelines } \\
\text { - Benefits/barriers/overcoming barriers }\end{array}$ & $\begin{array}{l}\text { 1. Resources: } \\
\text { - Pedometer } \\
\text { - Family dinner and physical activity planner (fridge magnet) } \\
\text { - 'Extra' food record sheet } \\
\text { - Session two information summary pamphlet } \\
\text { 2. Interactive activity: } \\
\text { - Playgroup } 10,000 \text { steps per day challenge }\end{array}$ \\
\hline 3 (Week 9) & $\begin{array}{l}\text { 1. Focus behaviour change: } \\
\text { - Review established short term goals } \\
\text { - Set new short term goals } \\
\text { 2. Focus physical activity: Muscle strength and flexibility exercises } \\
\text { - Guidelines } \\
\text { - Benefits/barriers/overcoming barriers }\end{array}$ & $\begin{array}{l}\text { 1. Resources: } \\
\text { - Muscle strength and flexibility exercise card (fridge magnet) } \\
\text { - Physical activity diary } \\
\text { - Session three information summary pamphlet } \\
\text { 2. Interactive activity: } \\
\text { - Muscle strength and flexibility exercises } \\
\text { - Integrated exercises " }\end{array}$ \\
\hline 4 (Week 13) & $\begin{array}{l}\text { 1. Focus behaviour change: } \\
\text { - Review established short term goals } \\
\text { - Set new short term goals } \\
\text { 2. Focus nutrition: } \\
\text { - Healthy eating messages } \\
\text { - Menu planning } \\
\text { - Food label reading } \\
\text { - Making sense of nutritional claims }\end{array}$ & $\begin{array}{l}\text { 1. Resources: } \\
\text { - Shopping list with healthy shopping tips } \\
\text { - Comparing packaged food per } 100 \mathrm{~g} \text { (fridge magnet) } \\
\text { - Session four information summary pamphlet } \\
\text { 2. Interactive activity: } \\
\text { - Reading packaged food labels } \\
\text { - Developing a daily menu }\end{array}$ \\
\hline 5 (Week 17) & $\begin{array}{l}\text { 1. Focus behaviour change: } \\
\text { - Review established short term goals } \\
\text { - Set new short term goals } \\
\text { - Overcoming relapses } \\
\text { 2. Focus nutrition: fats and sugars } \\
\text { - Recommended intake } \\
\text { - Benefits/barriers/overcoming barriers }\end{array}$ & $\begin{array}{l}\text { 1. Resources: } \\
\text { - Session five information summary pamphlet } \\
\text { 2. Interactive activity: } \\
\text { - Modifying recipes } \\
\text { - Healthy cooking methods }\end{array}$ \\
\hline 6 (Week 21) & $\begin{array}{l}\text { 1. Focus behaviour change: } \\
\text { - Review established short term goals } \\
\text { - Social support } \\
\text { 2. Focus nutrition: Fibre and Glycemic Index } \\
\text { - Recommended intake } \\
\text { - Benefits/barriers/overcoming barriers }\end{array}$ & $\begin{array}{l}\text { 1. Resources: } \\
\text { - Session six information summary pamphlet } \\
\text { 2. Interactive activity: } \\
\text { - Modifying recipes } \\
\text { - Healthy cooking methods }\end{array}$ \\
\hline
\end{tabular}




\section{Control group}

The control group participants will not receive any intervention materials. Their only contact with the project will include completing the questionnaires at the three data collection periods.

\section{Process measures \\ Participant process evaluation}

The REFRESH booklet will be assessed by the participants in terms of attractiveness, comprehension, personal relevance, believability, and legibility [48]. Workshop and staff feedback sheets will be provided to participants to assess the content and workshop delivery methods in the playgroup setting. Participants will be invited to comment on the REFRESH program's impact on their physical activity and nutrition behaviours and to provide suggestions for improvements to the intervention [49].

\section{Staff process evaluation}

The staff will provide feedback on the playgroup as a setting for health promotion programs. This evaluation will focus specifically on the playgroup characteristics, and the skills deemed necessary to deliver workshops in this setting. Staff will also provide feedback on working with mothers as a target group within the playgroup setting, what the mothers want to learn about nutrition and physical activity and common myths mothers report. Staff will also maintain a diary of their perceptions related to the delivery of the face-to-face workshop sessions, and responses by participants to the session content and activities.

\section{Outcome measures}

The self-administered questionnaire will be comprised of instruments which have been previously validated and tested for reliability [50-52], and will undergo further reliability testing prior to its use at baseline.

Physical activity will be measured by The International Physical Activity Questionnaire (IPAQ) [53]. This instrument has been accepted as the physical activity measurement tool in many settings and is specifically designed for population-based prevalence studies. Muscle strength exercise assessment will be based on recommendations from the American Heart Association and Australian physical activity guidelines [54]. Physical activity knowledge will be assessed by a modified version of the American Adult's Knowledge of Exercise Questionnaire [55]. Dietary intake will be measured using a modified version of the Fat and Fibre Barometer [56]. The New South Wales Government questionnaire will be used to measure soft drinks, fruit juice and snack consumption [57]. Added sugar consumption will be assessed using the 2005 National Health Interview Survey [58]. Nutrition knowledge will be assessed by a modified version of the General Nutrition Knowledge Questionnaire [59].
Self-efficacy for nutrition and physical activity behaviours will be assessed. Nutrition and physical activity self-efficacy will be assessed using items from previously validated instruments [52]. Validated questions will also confirm participants' stages of change regarding fruit and vegetable consumption [60]. Social support for physical activity will be assessed based on items from the Sallis et al. instrument [51].

General physical and mental health will be measured by The Medical Outcomes Study Short-Form Health Survey (SF-8) [61]. SF-8 is a standard international generic instrument of health status. It comprises two summary scales - the physical component summary (PCS) score and the mental component summary (MCS) score.

Demographic characteristics will include gender, age, educational level, country of birth, marital status, socioeconomic status, financial status and co-morbidities. Anthropometric measures will include self-reported height and weight, waist and hip girth. A recent study has confirmed that self report measures are suitable for such studies when a correction factor is applied [62].

Height, weight, waist and hip girth measurements will be conducted by research staff on a random subsample of 100 participants from the intervention group. Calculations of differences between self reported and research staff measured data will be undertaken to identify a correction factor based on the methodology of Dhaliwal et al. [62].

\section{Ethics}

The project protocol has been approved by the Curtin University Human Research Ethics Committee (approval number HR 186/2008).

\section{Discussion}

The REFRESH project is unique in using playgroups for a lifestyle intervention. The playgroup environment is an innovative setting for health promotion for mothers with young children, as it offers an exciting avenue to reach this target group and support behaviour change. The recruitment of participants through playgroups is beneficial as it will encourage all playgroup members to register for the program, thereby not just recruiting those who are motivated to adopt health enhancing behaviour [63].

The program will provide an opportunity for a variety of strategies to be implemented and evaluated. This evaluation data will be collected from participants in their own communities and not in a research centre, making the program relevant to the community based population and not just a clinical group. The project will provide guidelines for the development, implementation and evaluation of a minimal intervention home-based tailored physical activity and nutrition program. The information gathered will be valuable in helping to identify and address the barriers to participating in physical activity 
and nutrition programs for this target group. The project has the potential, to reduce chronic disease and enhance mental health for mothers of young children in the playgroup setting.

\section{Acknowledgements and funding}

The authors are grateful to the REFRESH project staff, Mr Steve Pratt, Dr Christina Pollard and Professor Brian Oldenburg for their contributions to the project. This study is funded by the Australian National Health and Medical Research Council (NHMRC) project grant.

\section{Author details}

${ }^{1}$ Western Australian Centre for Health Promotion Research, School of Public Health, Curtin University, Western Australia, Australia. ${ }^{2}$ Centre for Behavioural Research in Cancer Control, Curtin University, Western Australia, Australia. ${ }^{3}$ Curtin Health Innovation Research Institute, Curtin University, Western Australia, Australia. ${ }^{4}$ Griffith University and Mater Medical Research Institute, Queensland, Australia. ${ }^{5}$ Centre for Public Health Nutrition Research, University of Dundee, Dundee, UK.

\section{Authors' contributions}

SM coordinated the project, led the design of the REFRESH program and drafted the manuscript. JJ, PH, SB, CJ, SD, AM, AH and AA designed the study and revised the manuscript. All authors read and approved the final manuscript.

\section{Competing interests}

The authors declare that they have no competing interests.

Received: 8 July 2011 Accepted: 16 August 2011

Published: 16 August 2011

\section{References}

1. National Health Survey: Summary of results, Australia 2007-2008. [http:// www.abs.gov.au].

2. Rooney BL, Schauberger CW, Mathiason MA: Impact of perinatal weight change on long-term obesity and obesity-related illnesses. Obstet Gynecol 2005, 106:1349-1356.

3. Gunderson EP, Murtaugh MA, Lewis CE, Quesenberry CP, West DS, Sidney S: Excess gains in weight and waist circumference associated with childbearing: The Coronary Artery Risk Development in Young Adults Study (CARDIA). Int J Obes 2004, 28:525-535.

4. Soltani H, Fraser RB: A longitudinal study of maternal anthropometric changes in normal weight, overweight and obese women during pregnancy and postpartum. Br J Nutr 2000, 84:95-101.

5. Linne Y, Rossner S: Interrelationships between weight development and weight retention in subsequent pregnancies: the SPAWN study. Acta Obstet Gynecol Scand 2003, 82:318-325.

6. Siega-Riz AM, Viswanathan M, Moos MK, Deierlein A, Mumford S, Knaack J, Thieda P, Lux LJ, Lohr KN: A systematic review of outcomes of maternal weight gain according to the Institute of Medicine recommendations: birthweight, fetal growth, and postpartum weight retention. Am J Obstet Gynecol 2009, 201:339, e331-314.

7. Gore SA, Brown DM, West DS: The role of postpartum weight retention in obesity among women: a review of the evidence. Ann Behav Med 2003, 26:149-159.

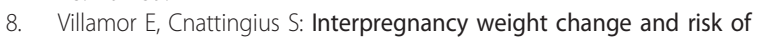
adverse pregnancy outcomes: a population-based study. Lancet 2006, 368:1164-1170.

9. Ramachenderan J, Bradford J, McLean M: Maternal obesity and pregnancy complications: A review. Aust and N Z J Obstet Gynaecol 2008, 48:228-235.

10. Ryan D: Obesity in women: a life cycle of medical risk. Int J Obes 2007, 31:S3-S7.

11. Ruager-Martin R, Hyde M, Modi N: Maternal obesity and infant outcomes. Early Hum Dev 2010, 86:715-722.

12. Nuss H, Freeland-Graves J, Clarke K, Klohe-Lehman D, Milani TJ: Greater nutrition knowledge Is associated with lower 1-year postpartum weight retention in low-income women. J Am Diet Assoc 2007, 107:1801-1806.
13. Oken E, Taveras EM, Popoola FA, Rich-Edwards JW, Gillman MW: Television, walking, and diet: associations with postpartum weight retention. Am J Prev Med 2007, 32:305-311.

14. Devine CM, Bove CF, Olson CM: Continuity and change in women's weight orientations and lifestyle practices through pregnancy and the postpartum period: the influence of life course trajectories and transitional events. Soc Sci \& Med 2000, 50:567-582.

15. Nash M: "You don't train for a marathon sitting on a couch": Performances of pregnancy 'fitness' and 'good' motherhood in Melbourne, Australia. Women's Studies International Forum 2010, 34:50-65.

16. Lewis B: Mothers reframing physical activity: family oriented politicism, transgression and contested expertise in Australia. Soc Sci \& Med 2005, 60:2295.

17. Nitzke S, Freeland-Graves J: Position of the American Dietetic Association: Total diet approach to communicating food and nutrition information. J Am Diet Assoc 2007, 107:1224-1232.

18. Webb DA, Bloch JR, Coyne JC, Chung EK, Bennett IM, Culhane JF: Postpartum physical symptoms in new mothers: their relationship to functional limitations and emotional well-being. Birth 2008, 35:179-187.

19. Singh AS, Mulder C, Twisk JWK, van Mechelen W, Chinapaw MJM: Tracking of childhood overweight into adulthood: a systematic review of the literature. Obes Rev 2008.

20. Williams LK, Veitch J, Ball K: What helps children eat well? A qualitative exploration of resilience among disadvantaged families. Hlth Edu Res 2011, 26:296-307.

21. Oken E: Maternal and Child Obesity: The Causal Link. Obstet Gynecol Clin North Am 2009, 36:361-377.

22. Hartman MA, Hosper K, Stronks K: Targeting physical activity and nutrition interventions towards mothers with young children: a review on components that contribute to attendance and effectiveness. Public Health Nutr 2010, FirstView:1-18.

23. Keller C, Records K, Ainsworth B, Permana P, Coonrod DV: Interventions for weight management in postpartum women. J Obstet Gynecol Neonatal Nurs 2008, 37:71-79.

24. Kuhlmann AKS, Dietz PM, Galavotti C, England LJ: Weight-management interventions for pregnant or postpartum women. Am J Prev Med 2008, 34:523-528.

25. Amorim AR, Linne $Y$, Lourenco PM: Diet, exercise, or both, for weight reduction in women after childbirth (review). Cochrane Database Syst Rev 2007.

26. National Institute for Health and Clinical Excellence: Dietary interventions and physical activity interventions for weight management before, during and after pregnancy. NICE: Public Health Guidance 272010.

27. Keller C, Allan J, Tinkle MB: Stages of change, processes of change, and social support for exercise and weight gain in postpartum women. $J$ Obstet Gynecol Neonatal Nurs 2006, 35:232-240.

28. Krummel DA, Semmens E, Boury J, Gordon PM, Larkin KT: Stages of change for weight management in postpartum women. J Am Diet Assoc 2004 104:1102-1108.

29. Boutron I, Moher D, Altman D, Schulz K, Ravaud P: Extending the CONSORT statement to randomized trials of nonpharmacologic treatment: explanation and elaboration. Ann Intern Med 2008, 148:295-309.

30. Pollock ML, Franklin BA, Balady GJ, Chaitman BL, Fleg JL, Fletcher B, Limacher M, Pina IL, Stein RA, Williams M, Bazzarre T: Resistance Exercise in Individuals With and Without Cardiovascular Disease: Benefits, Rationale, Safety, and PrescriptionAn Advisory From the Committee on Exercise, Rehabilitation, and Prevention, Council on Clinical Cardiology, American Heart Association. Circ J 2000, 101:828-833.

31. Egger G, Donovon R, Swinburn B, Giles-Corti B, Bull FC: Physical activity guidelines for Australians: Scientific background report. A report by the University of Western Australia and The Centre for Health Promotion and Research Sydney for the Commonwealth Department of Health and Aged Care. Canberra 1999.

32. Department of Health and Ageing: Food for health: dietary guidelines for Australians, a guide to healthy eating. Canberra 2005.

33. Playgroup Australia Australia Inc:. Annual Report. Perth 2010.

34. Playgroup Western Australia Inc.: Communities enriched through playgroups: Annual report 2009-2010. Perth 2010.

35. Australian Bureau of Statistics: Census of population and housing: socioeconomic indexes for areas (SEIFA) 2006, information paper. Information paper 17 Canberra: Australian Bureau of Statistics; 2008, 17. 
36. Thomas S, Reading J, Shephard RJ: Revision of the Physical Activity Readiness Questionnaire (PAR-Q). Can J Sport Sci 1992, 17:338-345.

37. Cohen J: Statistical power analysis for the behavioural sciences Hillsdale, NJ: Lawrence Erlbaum Associates; 1987.

38. Power Analysis Sample Size. [http://www.ncss.com/pass.html].

39. Coakes S, Steed L, Ong C: SPSS: analysis without anguish: version 17 for Windows Qld: John Wiley \& Sons Australia Ltd; 2010.

40. Glanz K, Rimer BK, Lewis FM: Health behaviour and health education: theory, research and practice San Francisco: Jossey-Bass; 2002

41. Prochaska JO, Diclemente CC: Stages and Processes of Self-Change of Smoking - toward an Integrative Model of Change. J Consult Clin Psychol 1983, 51:390-395.

42. Resnicow K, Dilorio C, Soet J, Borrelli B, Ernst D, Hecht J, Thevos A: Motivational interviewing in medical and public health settings New York: Guilford Press; 2002.

43. Walker LO: Managing excessive weight gain during pregnancy and the postpartum period. J Obstet Gynecol Neonatal Nurs 2007, 36:490.

44. Jones C, Burns S, Howat P, Jancey J, McManus A, Carter O: Playgroup as a setting for nutrition and physical activity interventions for mothes with young children: exploratory qualitative findings. Health Promot J Austr 2010, 21:92-98.

45. Howat P, Brown G, Burns S, MCManus A: Use of the PRECEDE-PROCEED Framework to guide planning and evaluation for health promotion Sydney: Oxford University Press; 2008

46. Green $L$, Kreuter $M,(E d s)$ : Health program planning: an educational and ecological approach. New York: McGraw Hill; 42005.

47. Walker L, Kim S, Sterling B, Latimer L: Developing health promotion interventions: a multisource method applied to weight loss among lowincome postpartum women. Public Health Nurs 2010, 27:188-195.

48. Burke L, Howat P, Lee A, Jancey J, Kerr D, Shilton T: Development of a nutrition and physical activity booklet to engage seniors. BMC Res Notes 2008, 1:1-7.

49. United States Department of Health and Human Services: Making health communication programs work; a planner's guide. Book Making health communication programs work; a planner's guide National Institutes of Health; 1992.

50. Forsén L, Nina Waaler L, Vuillemin A, Chinapaw MJM, van Poppel MNM, Mokkink LB, Willem van M, Terwee CB: Self-Administered Physical Activity Questionnaires for the Elderly: A Systematic Review of Measurement Properties. Sports Med 2010, 40:601-623.

51. Sallis J, Grossma R, Pinski R, Patterson T, Nader P: The development of scales to measure social support for diet and exercise behaviours. Prev Med 1987, 16:825-836.

52. Sallis J, Pinski R, Grossman R, Patterson T, Nader P: The development of self-efficacy scales for healthrelated diet and exercise behaviors. Health Educ Res 1988, 3:283-292.

53. Craig C, Marshall M, Sjostrom A, Bauman A, Booth B, Ainsworth M, Pratt U, Ekelund A, Yngve J, Sallis J, Oja P: International physical activity questionnaire: 12 country reliability and validity. Med Sci Sports Exer 2003, 35:1381-1395.

54. Haskell W, Lee I, Pate R, Powell K, Blair S, Franklin B, Macera C, Heath G, Thompson P, A B: Physical activity and public health: updated recommendations for adults from the American College of Sports Medicine and American Heart Association. Circ J 2007, 116:1-13.

55. Morrow JR, Krzewinski-Malone JA, Jackson AW, Bungum TJ, FitzGerald SJ: American adults' knowledge of exercise recommendations. Res Q Exerc Sport 2004, 75:231-237.

56. Wright J, Scott J: The fat and fibre barometer, a short food behaviour questionnaire: reliability, relative validity and utility. Aust J NutrDiet 2000, 57:33-39.

57. Flood VM, Webb K, Rangan A: Recommendations for short questions to asses food consumption in children for NSW Health Surveys City: NSW Centre for Public Health Nutrition; 2005.

58. Centre for Disease Control and Prevention: 2005 National Health Interview Survey. Book 2005 National Health Interview Survey National Center for Health Statistics; 2006.

59. Hendrie GA, DN C, Coveney J: Validtion of the General Nutrition Knowledge Questionnaire in an Australian community sample. Nutr and Diet 2008, 65:72-77.

60. Jalleh G, Lin C, Donovan RJ: Evaluation of the make smoking history 'Sugar 9Sugar' campaign: wave 23. Book Evaluation of the make smoking history 'Sugar Sugar' campaign: wave 23 Perth: Centre for Behavioural Research in Cancer Control, Curtin University; 2009.

61. Saris-Baglama R, Dewey C, Chisholm G, Chisholm B, Plumb E, King J,

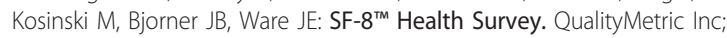
2007.

62. Dhaliwal SS, Howat P, Bejoy T, Welborn TA: Self-reported weight and height for evaluating obesity control programs. Am J Health Behav 2010, 34:489-499.

63. Treweek S, Pitkethly M, Cook J, Kjeldstrøm M, Taskila T, Johansen M Sullivan F, Wilson S, Jackson C, Jones R, Mitchell E: Strategies to improve recruitment to randomised controlled trials (Review). Cochrane Database Syst Rev 2010, 4

\section{Pre-publication history}

The pre-publication history for this paper can be accessed here: http://www.biomedcentral.com/1471-2458/11/648/prepub

\section{doi:10.1186/1471-2458-11-648}

Cite this article as: Monteiro et al:: The protocol of a randomized controlled trial for playgroup mothers: Reminder on Food, Relaxation, Exercise, and Support for Health (REFRESH) Program. BMC Public Health 2011 11:648

\section{Submit your next manuscript to BioMed Central and take full advantage of:}

- Convenient online submission

- Thorough peer review

- No space constraints or color figure charges

- Immediate publication on acceptance

- Inclusion in PubMed, CAS, Scopus and Google Scholar

- Research which is freely available for redistribution

Submit your manuscript at www.biomedcentral.com/submit
C Biomed Central 